\title{
Pengines: Web Logic Programming Made Easy
}

\author{
TORBJÖRN LAGER \\ University of Gothenburg, Sweden \\ Torbjorn.Lager@ling.gu.se \\ JAN WIELEMAKER \\ VU University Amsterdam, The Netherlands \\ J.Wielemaker@vu.nl \\ submitted 14 May 2014; revised 14 May 2014; accepted 27 March 2014
}

\begin{abstract}
When developing a (web) interface for a deductive database, functionality required by the client is provided by means of HTTP handlers that wrap the logical data access predicates. These handlers are responsible for converting between client and server data representations and typically include options for paginating results. Designing the web accessible API is difficult because it is hard to predict the exact requirements of clients. Pengines changes this picture. The client provides a Prolog program that selects the required data by accessing the logical API of the server. The pengine infrastructure provides general mechanisms for converting Prolog data and handling Prolog non-determinism. The Pengines library is small (2000 lines Prolog, 150 lines JavaScript). It greatly simplifies defining an AJAX based client for a Prolog program and provides non-deterministic RPC between Prolog processes as well as interaction with Prolog engines similar to Paul Tarau's engines. Pengines are available as a standard package for SWI-Prolog 7 11
\end{abstract}

KEYWORDS: web programming, query languages, agent programming, distributed programming

\section{Introduction}

Distributed systems play a central role in modern IT systems. We distinguish three different models for point-to-point communication between systems: (1) based on a query language (e.g., SQL or SPARQL), (2) based on a generic attribute-value exchange (e.g., HTTP) and (3) based on methods and datatypes (e.g., SunRPC, SOAP, CORBA, JSON-RPC). If Prolog is used as a component in such systems, the typical solution is to embed Prolog in another language through the foreign language interface. Alternatively, Prolog may be used to implement the wire protocol directly (often used for HTTP) or wrap a foreign library that implements the wire protocol (e.g., ODBC).

If the task of the Prolog-based component is simple and can easily be expressed as a deterministic function call, the above solutions are satisfactory. If, however, Prolog is used as a (deductive) database, the above is not ideal. In this scenario, an SQL or SPARQL like query language over the core relational predicates provided by the database is much more comfortable because it allows the client to specify a desired set of results in a uniform and flexible manner. Both SQL and

\footnotetext{
${ }^{1}$ A web-based demonstration of pengines is available at http://pengines.swi-prolog.org and can be downloaded from https://github.com/SWI-Prolog/pengines
} 
SPARQL provide (1) a language to express the desired result set, (2) a way to paginate result sets (offset and limit) and (3) a uniform access to data of different types (per column in SQL and the various RDF object types for SPARQL).

Without something similar to SQL or SPARQL, the Prolog server developer has to imagine all sensible ways to access the data based on the deterministic procedures and wrap these access functions in HTTP or some form of RPC. If the application is a-priori known and has a fairly fixed functionality, a top-down design can be satisfactory, but once this is not the case we will typically see a large and growing set of API functions with many options to select the proper data and represent it in a way that is suitable for the client application. This is where Pengines come in. The idea behind pengines is simple:

- A pengine is a thread on a (often remote) Prolog pengine server.

- The query language is Prolog, i.e., the client uploads a short Prolog program to the pengine that provides the data exchange needed by the client based on the clean relational interface of the deductive Prolog database.

- Subsequently, the client sends one or more Prolog queries with result templates to the pengine.

- The pengine answers with a set of answer tuples based on answer bindings of a template. Data representation is standardized. At the moment we have two formats: Prolog syntax for Prolog clients and a standard representation of Prolog terms in JSON for e.g., JavaScript clients.

- Pagination is based on Prolog backtracking. As an option, results can be batched in chunks of a certain size, e.g., return $(\max ) 20$ results per communication.

A pengine is closely related to Paul Tarau's logical engines (see section2). If a Prolog client is used, pengines can implement natural non-deterministic RPC (NDRPC) as well as coroutining. The JavaScript client allows for creating a pengine from Prolog embedded on the HTML page, sending the pengine a query and reacting on the 'answer events'.

This article is organized as follows. First we discuss related work, in particular the relation with logical engines as realised by Paul Tarau. Next, we informally introduce Pengines using a number of examples. In section 4 we describe the state machine used to realise Pengines and communicate with them as well as the core primitives. Section 7 describes a derived high level primitive (Prolog-RPC). Before the final conclusions section, we address security and future plans.

\section{Related work}

The notion of explicit Prolog engines has been explored extensively by Paul Tarau et al (Tarau and Majumdar 2009) in the context of the Jinni agent programming language and the BinProlog system. Tarau's engines are in-process and primarily designed to provide a clean alternative implementation for Prolog language constructs such as the all-solutions predicates (e.g., findall/3), exception handling as well as language constructs that are less common in the Prolog world, such as multiple coroutining blocks.

In contrast, pengines are designed primarily for creating and accessing Prolog engines on a remote server and communicating with them using multiple languages (currently Prolog and JavaScript). Tarau's engine API and the Prolog implementation of our Pengine client are closely related. We explain the differences below. 
Creation Tarau's engines are created using a Goal and AnswerTemplate. As our pengines typically run remote, they are more heavy weight and we decided that a created pengine can be used to execute multiple queries using pengine_ask/3, i.e., a pengine is an engine that runs a goal that asks for goals to execute. Typically, a pengine is created with a Prolog source, providing the code to execute. This is irrelevant for Tarau's engines as they run in the same process. (Remote) pengine creation requires some additional information, such as the address of the server and the data-format for exchanging events (Prolog or JSON).

Yield/return Tarau's yield and return primitives are represented using pengine_output/1 (return) and pengine_input/2 (yield).

One-sided communication Designed to run over HTTP, only the 'client' can take initiative in the pengines world. The pengine_pull_response/2 primitive realises bi-directional initiative, based on the 'long polling' technique that is commonly used in the context of HTTP to achieve server initiative. This is not needed if engines are embedded in the same process or can use a bi-directional communication channel. (See also section 12, )

Pengines can also be regarded as a high-level interface to Prolog, similar to InterProlog (Calejo 2004) and the multi-language interface supported by ECLIPSE Prolog (Shen et al. 2002). Whereas InterProlog provides a Java-Prolog interface, ECLIPSE supports Tcl/Tk and Visual Basic as well as Java. At this time, Pengines only supports Prolog-Prolog and JavaScript-Prolog communication, the latter of utmost importance for a seamless integration between Prolog and the Web. Other languages can easily be added. Pengines also handles backtracking over nondeterministic queries as well as I/O, something which is not supported by the other interfaces.

\section{Pengines by example}

We proceed to give a number of examples showing how pengines can be created and controlled from any Prolog program, or from JavaScript running in a web browser. We also show how to make non-deterministic remote procedure calls (NDRPC) using a predicate implemented on top of the Pengines core predicates.

\subsection{Prolog interacting with a pengine}

In this example we load the Pengines library, use pengine_create/1 to create a pengine in a remote pengine server, and inject a number of clauses in it. We then use pengine_event_loop/2 to start an event loop that listens for three kinds of event terms. Running main/0 will write the terms $q(a), q(b)$ and $q(c)$ to the standard output of the local process. Using pengine_ask/ 3 with the option template $(\mathrm{X})$ would produce the output $\mathrm{a}, \mathrm{b}$ and $\mathrm{c}$. Removing the server ('http://pengines.org') option would solve the query $q(X)$ locally instead, although still concurrently.

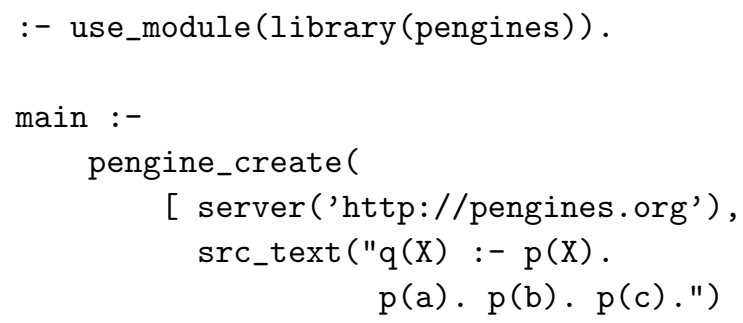




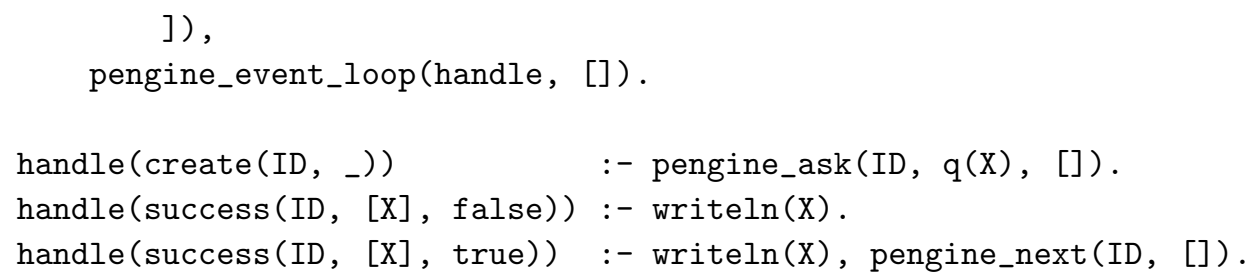

\subsection{JavaScript interacting with a pengine}

In this example, we show how to create and interact with a pengine from JavaScript. Loading the page brings up the browser's prompt dialog, waits for the user's input, and writes that input in the browser window. If the input was 'stop', it stops there, else it repeats $2^{2}$ Note that $\mathrm{I} / \mathrm{O}$ works as expected. All we need to do is to use pengine_input/2 instead of read/1 and pengine_output/1 instead of write/1.

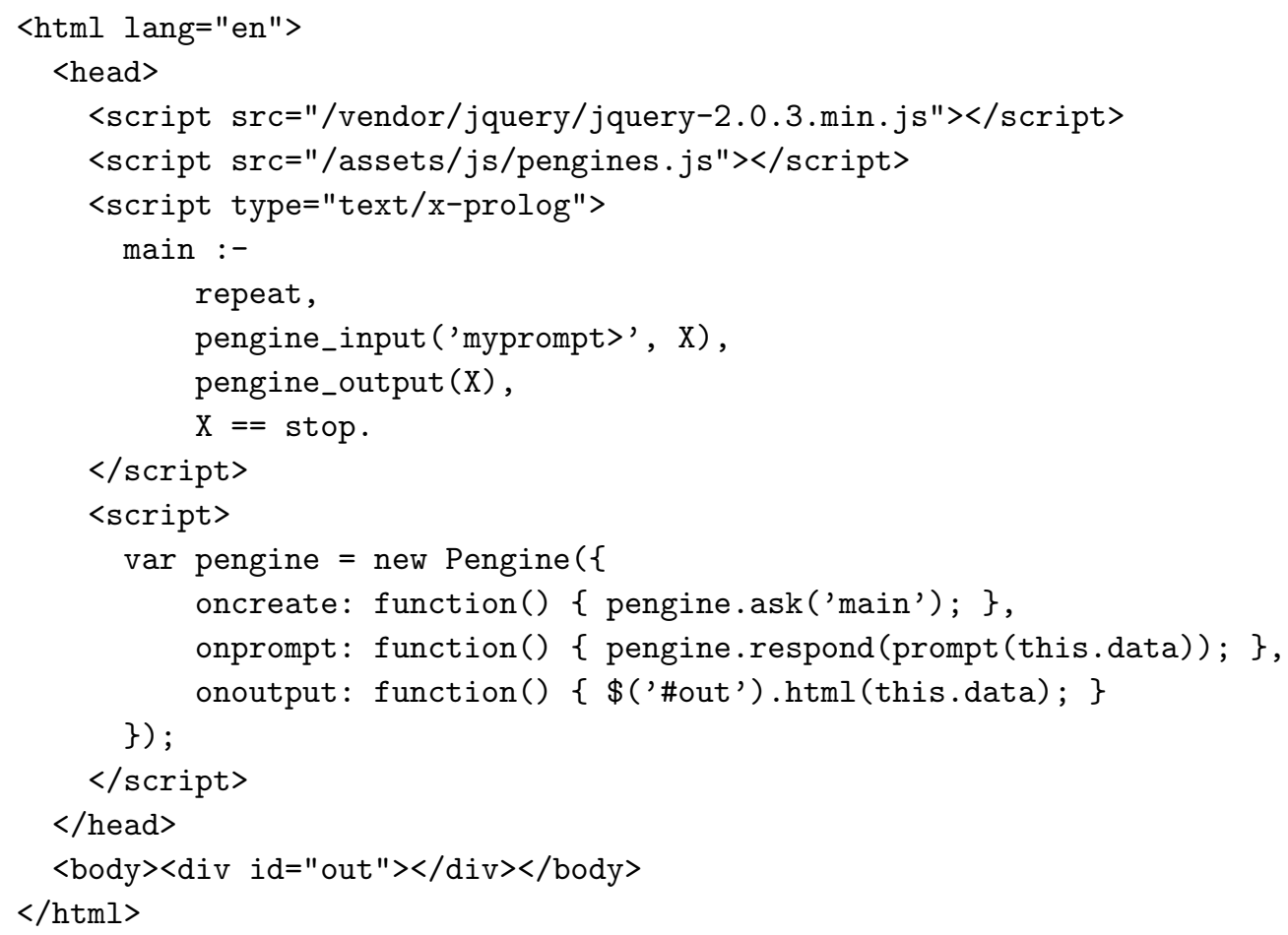

\subsection{Pengines non-deterministic RPC}

Our third example shows that a non-deterministic predicate can be called remotely by means of pengine_rpc/2, yet behave exactly as if called locally:

?- $\operatorname{member}(\mathrm{X},[\mathrm{a}, \mathrm{b}, \mathrm{c}, \mathrm{d}])$,

pengine_rpc('http://pengines.org', $p(X)$,

\footnotetext{
${ }^{2}$ We could have implemented main/0 as a recursive loop instead, but using a repeat-fail loop nicely serves to demonstrate that Pengines gives the programmer the same options as when programming against a simple command-line shell.
} 


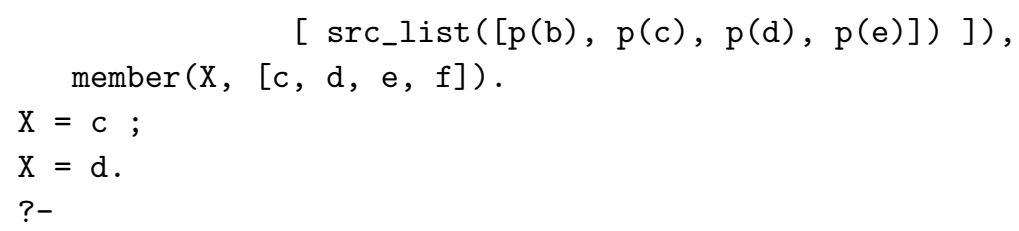

In this case, using the src_list option, we choose to inject four $\mathrm{p} / 1$ clauses in the pengine on creation time. There are other similar options available: the src_text option will consult a string of Prolog text instead (see the example in 3.1p, the src_url option will consult the file referred to by a URL, and the src_predicate option will simply carry over the predicates referred to by a list of predicate indicators to the pengine and assert them there as well.

\subsection{Using pengines as a semantic web query language}

Our last example is a realistic example that illustrates how pengines can be used to make deductive databases available over the web. We build this example on top of an existing demo application for the semantic web. The core of the semantic web is the data description language $\mathrm{RDF}^{3}$ An RDF model consists of a set of triples of the form $\{$ Subject, Predicate, Object $\}$. The ClioPatria system 4 provides a storage layer for RDF that makes the RDF triples available using the declarative relation $\mathrm{rdf} / 3$.

This example illustrates the following benefits from pengines: (1) adding pengines provides a generic API to the application with only a few lines of code and (2) available relations on the server can be combined using Prolog control structures that are executed on the server.

The short code fragment below adds pengine support to ClioPatria. After loading the pengines service, we load the RDF libraries into the pengine context to make them accessible. Next, we need to tell the sandboxing library that the predicate $\mathrm{rdf} / 3$ is safe, i.e., executing it does not pose a security risk.

:- use_module(library(pengines)).

:- use_module (pengine_sandbox:library (semweb/rdf_db)).

:- use_module (pengine_sandbox:library (semweb/rdfs)).

:- use_module (library (sandbox)) .

:- multifile sandbox:safe_primitive/1.

sandbox:safe_primitive $\left(r d f \_d b: r d f\left({ }_{-},{ }_{-}\right)\right)$.

For the remainder of this example, we populate ClioPatria with the 'Open Piracy' dat 55 The Open Piracy dataset contains information about piracy events around the world. The central notion is an 'event' that has a type, involved actors, a place and a time. We will use pengine_rpc/3 to query the ClioPatria service about events. First, we define simple building blocks for querying the dataset, that (1) relate an event to its position on the globe and (2) test that the event takes place in a given bounding box on the globe. Note that the code below could be loaded a priori into the ClioPatria instance providing a high-level domain specific query language, or can be uploaded from the client.

3 http://www.w3.org/TR/2004/REC-rdf-primer-20040210/

4 http://cliopatria.swi-prolog.org

5 http://cliopatria.swi-prolog.org/help/source/doc/home/vnc/prolog/src/ClioPatria/web/ tutorial/Piracy.txt 


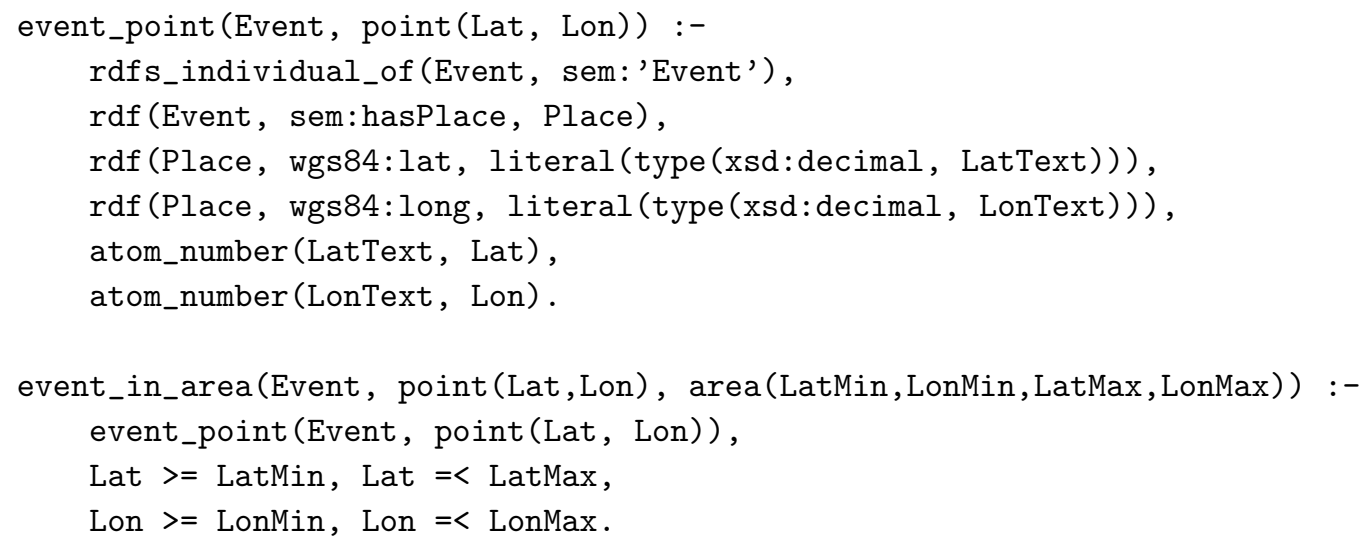

Given the code above, the client can query for events in a given bounding box using the code below. The src_predicates option transfers the given predicates from the client to the pengine on the server.

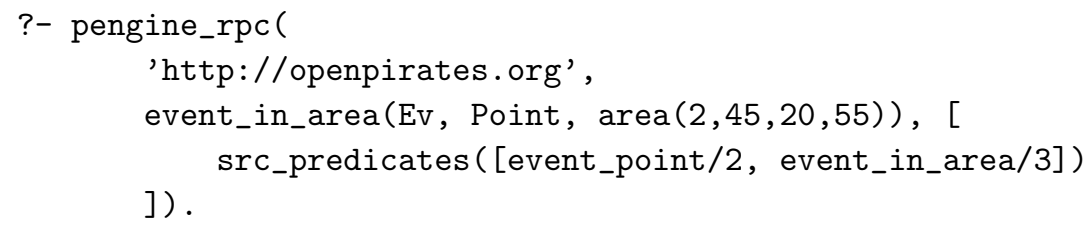

Note that the execution of the event_in_area/3 predicate takes place on the server. The execution consists of a conjunction of several $\mathrm{rdf} / 3$ goals, data transformation and conditions, yet only results are passed back to the client.

\section{The Prolog Transport Protocol}

Underlying the design of the Pengines package is an analysis of the conversations taking place between Prolog and a user (which could be a human or another piece of software). Such conversations follow a communication protocol that we refer to as the Prolog Transport Protocol (PLTP). The protocol is based on the Prolog 4-port model, (Byrd 1980) extended with exceptions and $\mathrm{I} / \mathrm{O}$ and has been modelled by means of so called communicating finite-state machines (Brand and Zafiropulo 1983). A slight modification of the protocol, referred to as PLTP ${ }_{H T T P}$, makes it compatible with HTTP, where only the client can take initiative. Figure 1 depicts the communicating finite-state machines for PLTP ${ }_{H T T P}$ and HTTP. Labels in bold indicate requests, and labels with a slash in front indicate responses.

Figure 2 shows the traffic between a client and a server on which a pengine (with ID 1234) is first created, then used to solve queries involving both backtracking and $\mathrm{I} / \mathrm{O}$, and finally destroyed.

\section{The Prolog client: Core predicates}

We distinguish the pengine core predicates from derived predicates defined in terms of the core. The core predicates are listed in the left column of Table 1 . We deal with some of the derived predicates in Section 7 . 


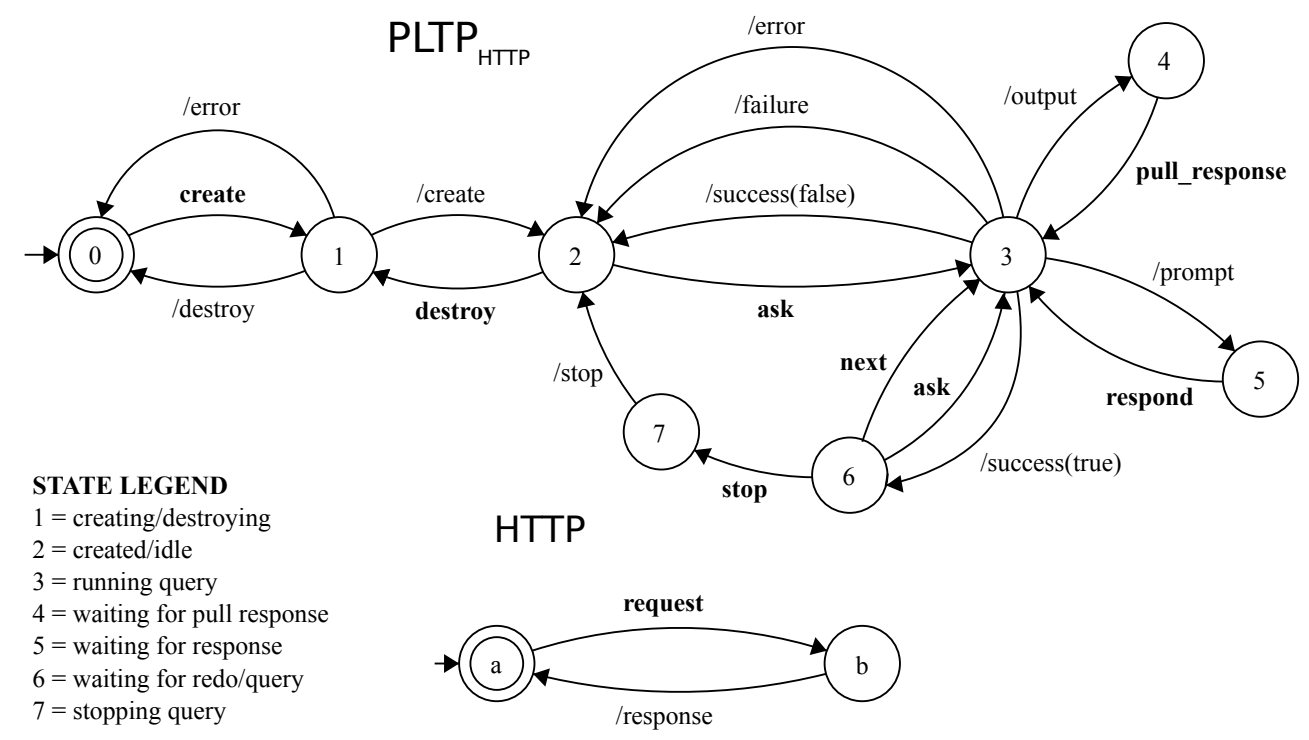

Fig. 1. Communicating finite-state machines for PLTP ${ }_{H T T P}$ and HTTP

Each core predicate corresponds to a request in the PLTP protocol (see the transitions with labels set in bold in Figure 11). The terms listed in the right column of Table 1 are returned as responses to such requests.

Table 1. Prolog requests and responses. Requests result in a single response, but the response depends on the program behaviour. Figure 1 defines which responses are possible for each request.

\begin{tabular}{ll}
\hline \hline Requests & Responses \\
\hline pengine_create(Options) & create(ID, Data) \\
pengine_ask(ID,Query, Options) & output (ID, Data) \\
pengine_pull_response(ID) & prompt (ID, Data) \\
pengine_respond(ID, Term) & success (ID, Data, More) \\
pengine_next(ID) & failure (ID) \\
pengine_stop(ID) & error(ID,Data) \\
pengine_destroy(ID) & stop(ID) \\
pengine_abort(ID) & destroy(ID)
\end{tabular}

Several pengine core predicates accept options that modify or control the creation or run time behaviour of pengines. We describe those that we believe are the most important ones. For pengine_create/1 there is an option id(-ID) that will bind ID to a UUID that can be passed around and that allows any process that knows it to enter into a conversation with the pengine. The name (+Atom) option allows a master process to name its slave pengines, and to use simple user defined names instead of opaque system generated identifiers in future conversations. When a process creates a pengine remotely, with a request over HTTP, it receives the identifier in the response. In order to save network round-trips, Prolog code in the form of a list of clauses, Prolog text, or a URL resolving to ${ }^{*}$.pl file accessible on the Web can be injected into the pengine at creation time. 


\begin{tabular}{|l}
\multicolumn{1}{c|}{ Shell } \\
\$ swipl \\
?- member(X, [a, b]). \\
X=a; \\
X=b. \\
?- write(hello). \\
hello \\
true. \\
?- read(X). \\
|: hello. \\
X= hello. \\
?- halt. \\
\$
\end{tabular}

\begin{tabular}{ll} 
pengine_create([]) \\
\hline create(1234, true) \\
\hline pengine_ask(1234, member(X, [a, b]), [template(X)]) \\
\hline puccess(1234, [a], true) \\
\hline puccess(1234, [b], false) \\
\hline pengine_ask(1234, pengine_output(hello)) \\
\hline pengine_pull_response(1234) \\
\hline success(1234, hello) \\
\hline pengine_ask(1234, [pengine_output(hello)], false) \\
\hline prompt(1234, 'l:') \\
\hline pengine_respond(1234, hello) \\
\hline success(1234, [hello], false)) \\
\hline pengine_destroy(1234) \\
\hline destroy(1234) \\
\hline
\end{tabular}

Fig. 2. The PLTP run (on the right) corresponding to a user's interaction with Prolog (on the left). ' 1234 ' is the Pengine's identifier, which is a UUID in the actual implementation.

The pengine_ask/3 predicate also accepts options. The template (+Template) option allows the invoker of a query to select the interesting parts of solutions through variables shared with the query. A list of instantiations of Template (rather than a list of instantiations of the whole query) will appear in the second argument of the success event terms returned to the invoker by the pengine. The chunk $(+\mathrm{N})$ option allows the invoker to retrieve solutions in chunks of $\mathrm{N}$ from the pengine, rather than the default one by one. As we shall see in section 8 , this mechanism can be used not only to paginate the solutions generated by a query to (say) a deductive database, but also to optimise performance by allowing to make fewer network round-trips.

\section{The JavaScript client}

The JavaScript client code consists of a definition of a single Pengine object, written in less than 150 lines of JavaScript. When an object is created, a pengine is (by default) created on the pengine server from which this client code was loaded. The object exposes a number of methods that allows the client to send requests (queries, input, commands, etc.) to the pengine. By default, pengine responses reach the client in the form of JSON events to be handled by JavaScript functions. Table 2 lists the main methods exposed by the JavaScript object, as well as the form of JSON responses. 
Table 2. JavaScript requests and responses

\begin{tabular}{ll}
\hline \hline Requests & Responses \\
\hline pengine = new Pengine(options) & \{"event":"create", "id":ID, "data":Data $\}$ \\
pengine.ask(query, options) & $\{$ "event":"output", "id":ID, "data":Data $\}$ \\
pengine.pull_response(ID) & $\{$ "event": "prompt", "id":ID, "data":Data $\}$ \\
pengine.respond(term) & $\{$ "event": "success", "id":ID, "data":Data, "more":Bool $\}$ \\
pengine.next() & $\{$ "event":"failure", "id":ID \\
pengine.stop() & $\{$ "event":"error", "id":ID, "data":Data $\}$ \\
pengine.destroy() & $\{$ "event":"stop", "id":ID \\
pengine.abort() & $\{$ "event":"destroy", "id":ID \\
\hline \hline
\end{tabular}

For data, Pengines defines the following mapping between ground Prolog terms and JSON ${ }^{6}$

(i). A Prolog atom is mapped to a JSON string.

(ii). A Prolog number is mapped to a JSON number.

(iii). A Prolog list is mapped to a JSON array.

(iv). The Prolog terms @(true), @(false) and @(null) are mapped to the JSON constants true, false and null, respectively.

(v). A Prolog term json (NameValueList), where NameValueList is a list of Name=Value pairs, is mapped to a JSON object.

(vi). Any other complex Prolog term T is mapped to a JSON object of the form "functor" : $\mathrm{F}$, "args": $\mathrm{A}$ \} where $\mathrm{F}$ is a string representing the functor of $\mathrm{T}$ and $\mathrm{A}$ is the list of JSON values representing T's arguments.

For example, the JSON response encoding the second solution to append (Xs, Ys, [a , b , c] ) is:

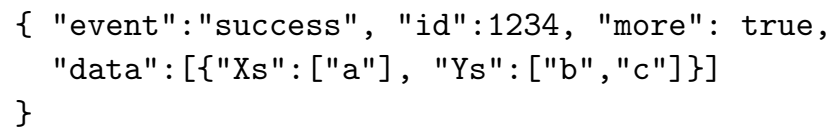

\section{The Prolog client: Derived predicates}

The core pengine predicates are deterministic, yet they can control one or more pengines solving possibly non-deterministic queries. The package also offers a number of derived nondeterministic predicates, built on top of the deterministic ones, that can solve queries "the Prolog way", binding query variables in the process, backtracking for more solutions. Of these predicates, pengine_rpc/3 is the most important. By means of pengine_rpc/3 a pengine running in a pengine server $\mathrm{A}$ can call and try to solve a query in the context of another pengine server $\mathrm{B}$, taking advantage of the data being offered by $\mathrm{B}$, just as if the data was local to $\mathrm{A}$.

Implementing pengine_rpc/3 involves creating a remote pengine, which may send additional predicates to the remote server and subsequently realise the normal declarative and operational semantics of running a Prolog predicate by acting on the pengine events.

6 This mapping will be reconsidered with the introduction of strings and dicts in SWI-Prolog version 7, see http: //www.swi-prolog.org/pldoc/man?section=extensions 


\section{Efficiency considerations}

Efficiency considerations when programming with pengines are important. There are two major sources of inefficiency related to network programming: network latency and bandwidth. Bandwidth requirements are limited by bringing the code to the data. E.g., consider solving $\mathrm{p}(\mathrm{X}, \mathrm{Y}), \mathrm{q}(\mathrm{Y}, \mathrm{Z})$ where $Y$ is a large intermediate result that is not needed by the client. By default, Pengines will transport all variable bindings and thus also $Y$. This can be avoided in one of two ways. Either we can define a predicate $\mathrm{pq}(\mathrm{X}, \mathrm{Z})$, or we can use the option template $(\mathrm{Z})$ to only transport the binding for $Z$.

The impact of network latency is limited by reducing the number of round trips. This is achieved with three features. First of all, the first query may be passed with the create command. Second, the pengine by default self-destroys itself on determinstic completion, failure or error of the initial query. Third, the chunk $(\mathrm{N})$ option, sends answers in chunks of the specified size. Calling pengine_rpc(URL, Query, $[\operatorname{chunk}(\mathrm{N})]$ ) will result in a call to find_n(N, Query, Query, Solutions) ${ }^{7}$ on the pengine server located at URL.

\section{Security}

There are three layers of safety that relate to pengines. First of all, we can rely on the safety of the underlying HTTP protocol. Considering that Prolog has full access to the OS, this is like using an outdated unencrypted telnet session to the client. Alternatively, we can use HTTPS with authentication, which makes it similar to SSH access to a shell.

To facilitate anonymous users, SWI-Prolog provides library(sandbox) ${ }^{8}$ which exports safe_goal/1. This predicate performs abstract interpretation of the argument goal and either succeeds, throws an instantiation_error if it cannot prove sufficient instantiation to a meta-goal or throws a permission_error if it encounters a possibility that a predicate may be called that is not white-listed. The library provides a predefined whitelist consisting of pure Prolog built-in predicates, which enables it to prove the safety of many of the pure Prolog libraries.

The above is typically sufficient if the server uses plain Prolog. If it uses, e.g., the SWI-Prolog RDF database, the C-defined query facilities of this library can be added to the whitelist.

Finally, by means of various application settings the Pengines library also offers some protection against denial of service (DoS) attacks. One setting determines the number of pengines that can be run simultaneosly by an application, another setting the maximum number of slave pengines that a master process may create, and a third setting is a timeout that aborts the computation after a set time, thus protecting against runaway computations.

\section{Portability and interoperability}

The current implementation of pengines is heavily based on support for multiple threads as well as the SWI-Prolog HTTP server and client libraries (Wielemaker et al. 2008). It can probably be ported fairly easily to YAP. Porting to other Prolog implementations that implement the ISO working draft for threads 9 is probably feasible if the systems provide HTTP server and client

\footnotetext{
7 find_n(+N, ?Template, +Goal, ?List) acts like findall/3 but returns only the first $\mathrm{N}$ bindings of Template to List, on backtracking another batch of $\mathrm{N}$ bindings, and so on

8 http://www.swi-prolog.org/pldoc/doc/swi/library/sandbox.pl

9 http://logtalk.org/plstd/threads.pdf
} 
access. It is also possible to realise the protocol using Prolog processes managed from a conventional HTTP server. Such a coarse grain implementation is more robust against malicious pengines, but individual pengines start much slower and cannot easily share a Prolog database.

To achieve maximal interoperability between pengine platforms, that will allow a client on one platform to send and execute code on another platform, an effort must be made to standardise a subset of Prolog that runs in the same way on all platforms. Fortunately, such an effort only needs to deal with a safe subset of Prolog (see Section 9 ).

\section{Evaluation}

In this section we present insight into the overhead involved in using pengines. Table 3 shows the basic HTTP overhead, a minimal pengine RPC call and the execution time of the example from section 3.4 in three scenarios. We minimised network overhead by using connections to localhost. Timings were performed on a machine with an Intel Core i7-3770 CPU running Ubuntu 13.10 (kernel 3.11) and SWI-Prolog 7.1.14. Times are in milliseconds, taking the average of 10 runs, each with 1,000 iterations.

Table 3. Basic timing

\begin{tabular}{lrr}
\hline \hline Test & CPU time (ms) & Wall time (ms) \\
\hline Most simple HTTP request & 0.4 & 0.8 \\
RPC for true & 0.9 & 1.9 \\
event_in_area/3 on server & 7 & 7 \\
RPC event_in_area/3, chunk $=1$ & 159 & 386 \\
RPC event_in_area/3, chunk $=128$ & 11 & 39 \\
\hline \hline
\end{tabular}

The effect of chunking the result set is significant because fetching the next result batch involves an HTTP request. Nevertheless, using a high value may not be the optimal choice if not all answers are needed. Figure 3 illustrates the elapsed and CPU time used for fetching all 1981 solutions for event_point/ 2 with different chunk sizes.

\section{Future plans}

The concept of pengines is simple and is not likely to change much. One addition that we do however contemplate is a way to allow two pengines that are created and "owned" by different masters to communicate. Currently, this is not possible.

Two areas need further attention: security and performance. The current security model aims at retrieving data from the server without compromising it. Future versions are likely to include support to deny access to certain data and provide authorized access to certain data, which may include update operations. Regarding performance, it is easy to get into scenarios where the HTTP protocol and network connection overhead become severely limiting factors, in particular for pengine_rpc/3. Protocol and connection overhead can be reduced by using WebSockets ${ }^{10}$ We

\footnotetext{
10 http: //www . websocket .org/
} 


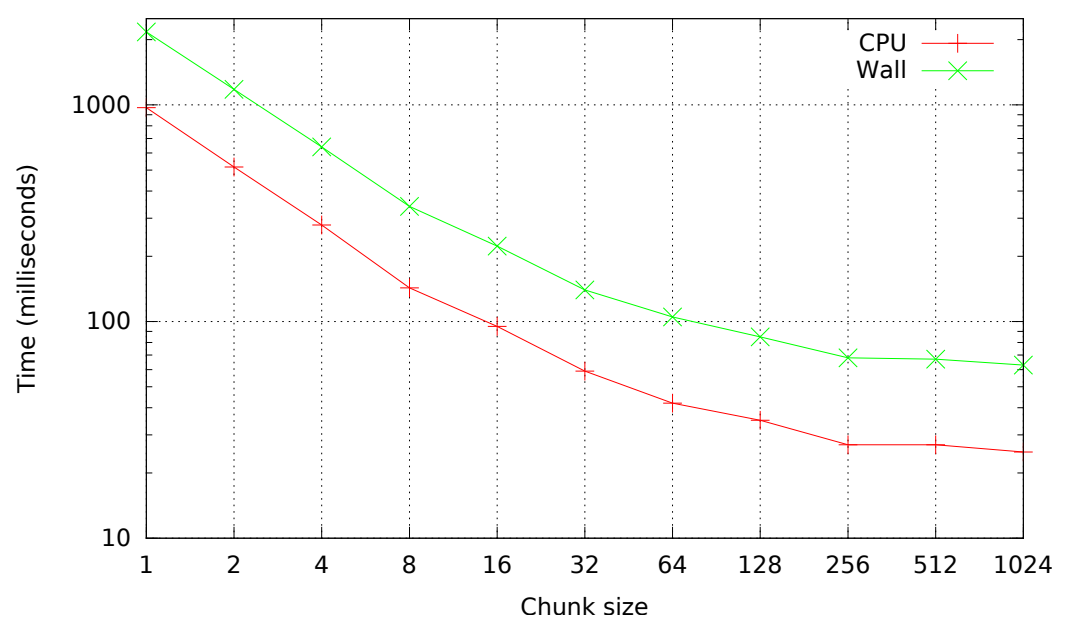

Fig. 3. Performance with varying chunk sizes

also plan to implement dynamic chunking of the results. This could either be the pengine_rpc/3 client dynamically switching to larger chunks or the server computing additional answers while waiting for a next command and returning all available answers when the next arrives.

\section{Conclusions}

Pengines extend Tarau's Prolog engines by allowing for Prolog engines to live in a remote (Pro$\log$ ) process. The communication with pengines is based on a state machine and uses either Prolog or JSON for serialization of messages. The current transport protocol is HTTP, which facilitates web applications and allows pengines to communicate smoothly with common firewall configurations. Prolog interaction with pengines can be used to implement agent interaction protocols, control structures such as coroutining blocks as well as convenient non-deterministic Prolog RPC. JSON based interaction constitutes an ideal way to interface Prolog with JavaScript for implementing web applications.

Creating a remote pengine may involve uploading a Prolog program to the server. 'Bringing the code to the data' greatly reduces data exchange between client and server. It allows the server to implement only a clean relational interface, while the client can ask questions that involve multiple relations efficiently.

Pengines are versatile. Applications include providing a web interface for a classical question/response Prolog application, complex network transparent control structures, Prolog-toProlog RPC, a modular alternative to the SPARQL query language for the semantic web or a web application based on a Prolog server.

We would like to stress that Pengines is not just yet another library for SWI-Prolog. We believe our work can be viewed more abstractly, as a description of a general approach to web logic programming, that can be given a concrete manisfestation not only for Prolog but also for other one-tuple-at-a-time logic programming languages. 


\section{References}

Brand, D. AND Zafiropulo, P. 1983. On communicating finite-state machines. Journal of the ACM (JACM) 30, 2, 323-342.

BYRD, L. 1980. Understanding the control flow of Prolog programs. In Logic Programming Workshop, S. Tarnlund, Ed. Debrecen, Hungary, 127-138.

CALEJO, M. 2004. Interprolog: Towards a declarative embedding of logic programming in java. In JELIA, J. J. Alferes and J. A. Leite, Eds. Lecture Notes in Computer Science, vol. 3229. Springer, 714-717.

Shen, K., Schimpf, J., Novello, S., AND Singer, J. 2002. A high-level generic interface to external programming languages for eclipse. In $P A D L$, S. Krishnamurthi and C. R. Ramakrishnan, Eds. Lecture Notes in Computer Science, vol. 2257. Springer, 262-279.

TARAu, P. AND Majumdar, A. 2009. Interoperating logic engines. In Practical Aspects of Declarative Languages. Springer, 137-151.

Wielemaker, J., Huang, Z., And Van der MeiJ, L. 2008. Swi-prolog and the web. Theory and Practice of Logic Programming 8, 3, 363-392. 